Nonlinear Processes in Geophysics, 12, 441-450, 2005

SRef-ID: $1607-7946 / \mathrm{npg} / 2005-12-441$

European Geosciences Union

(C) 2005 Author(s). This work is licensed

under a Creative Commons License.

\title{
Nonlinear compressional electromagnetic ion-cyclotron wavepackets in space plasmas
}

\author{
I. Kourakis ${ }^{1,2}$ and P. K. Shukla ${ }^{1}$ \\ ${ }^{1}$ Institut für Theoretische Physik IV, Fakultät für Physik und Astronomie, Ruhr-Universität Bochum, 44780 Bochum, \\ Germany \\ ${ }^{2}$ Max-Planck-Institut für extraterrestrische Physik, Giessenbachstrasse, 85740 Garching, Germany
}

Received: 8 February 2005 - Revised: 11 March 2005 - Accepted: 11 March 2005 - Published: 7 April 2005

\begin{abstract}
The parametric coupling between large amplitude magnetic field-aligned circularly polarized electromagnetic ion-cyclotron (EMIC) waves and ponderomotively driven ion-acoustic perturbations in magnetized space plasmas is considered. A cubic nonlinear Schrödinger equation for the modulated EMIC wave envelope is derived, and then solved analytically. The modulated EMIC waves are found to be stable (unstable) against ion-acoustic density perturbations, in the subsonic (supersonic, respectively) case, and they may propagate as "supersonic bright" ("subsonic dark", i.e. "black" or "grey") type envelope solitons, i.e. electric field pulses (holes, voids), associated with (co-propagating) density humps. Explicit bright and dark (black/grey) envelope excitation profiles are presented, and the relevance of our investigation to space plasmas is discussed.
\end{abstract}

\section{Introduction}

Long wavelength left and right-hand polarized electromagnetic ion-cyclotron (EMIC) waves are common in the Earth's magnetosphere (Stix, 1992; Baumjohann, 1996), auroral ionosphere, as well as in the solar corona (Cranmer et al., 1999). Large amplitude EMIC waves play a very important role in energizing and heating ions, in addition to creating large scale density and magnetic field perturbations in space and heliospheric plasmas. EMIC wave-related modulated structures associated with density perturbations were often observed during satellite missions, e.g. FAST (McFadden et al., 1998; Cattell et al., 1998; Chaston et al., 2002), Freja (Stasiewicz et al., 2000) and Cluster (Meziane, 2004a, b) in the Earth's magnetosphere. Since EMIC waves are involved in a wide variety of physical processes, they have been the object of many publications, regarding theory (Lee, 1972; D'Angelo, 1977; Praburam et al., 1990; Gomberoff, 1996; Lund and LaBelle, 1997; Huddleston et al., 1997;

Correspondence to: I. Kourakis

(ioannis@tp4.rub.de)
Guglielmi et al., 1999) and space observations (McFadden et al., 1998; Cattell et al., 1998; Chaston et al., 2002; Stasiewicz et al., 2000; Meziane et al., 2004a, b; also see Mazelle et al., 2004, and references therein), as well as EMIC-related laboratory experiments (Alport et al., 1983, 1994; Alport and van Niekerk, 1990). Furthermore, EMIC waves are also important in fusion plasmas (Greene and Gould, 1991) and in isotope separation studies, where their resonance with ion species is employed as an effective heating mechanism (ioncyclotron resonance heating), in order to sustain the burning thermonuclear plasma state (ITER Physics Expert Group, 1999). Finally, Landau resonance interactions between dispersive Alfvén/electromagnetic ion-cyclotron waves were invoked as a mechanism for the solar coronal electron heating (see e.g. in Li et al., 1999; Hu and Habbal, 1999; Shukla et al., 2004).

In a generic manner, as the wave amplitude increases, nonlinear effects become important. One such effect, which is long known to govern wave propagation in dispersive media, is the nonlinear modulation of the carrier wave amplitude due to the parametric coupling with non-resonant low-frequency perturbations. Following the early work of Hasegawa (1970, 1972), who used a reductive perturbation method for studying the amplitude modulation of electromagnetic electroncyclotron (EMEC) waves by low-frequency magnetohydrodynamic (MHD) perturbations, Karpman and Washimi (Karpman and Washimi, 1976) included the ponderomotive force in the description of the modulation of EMEC waves by low-frequency magnetoacoustic perturbations. Those results were then applied by the same authors to study the amplitude modulation of high-frequency magnetic field-aligned electron cyclotron waves due to their coupling with slow magnetosonic waves (Karpman and Washimi, 1977; Karpman and Stenflo, 1988), within a MHD approximation, and the technique was later employed by Shukla and co-workers (Shukla and Stenflo, 1984, 1985; Rao and Shukla, 1997, 1998) in a description of the parametric interactions between whistlers and ion-acoustic waves. Recently, the formalism was adopted as a model for the localized whistler-related 
envelope structures coupled to density perturbations (Eliasson and Shukla, 2004; Kourakis and Shukla, 2005) (whistler bi-solitons, or whistlerons), which are frequently observed in the Earth's magnetosphere. The method was recently also employed in a study of pair (e.g. electron-positron) plasmas (Stenflo et al., 1985; Cattaert et al., 2005), which are believed to exist in pulsars and active galactic nuclei (AGN).

From a fundamental point of view, the ponderomotive coupling between a high-frequency field and driven low frequency density perturbations results in a modulation of the amplitude of the former, which then essentially drives the latter in a forcing manner. The generic modulation formalism employed in the study of this mechanism is related to the phenomenon of localisation via modulational instability, which possibly leads to the formation of envelope excitations, i.e. breather-like envelope pulses or holes (voids). These excitations are intrinsically different from the nontopological solitons (pulses) obtained via the Korteweg-de Vries (KdV) small-amplitude nonlinear theory. An interested reader may refer to Grimshaw (2005) (also to Fedele, 2002; Fedele and Schamel, 2002) for a critical discussion of the relation between the nonlinear envelope soliton formalism, and the modified $\mathrm{KdV}$ (mKdV) (also KdV, respectively) equation(s), on the other.

In this paper, we are interested in studying parametric interactions between large amplitude magnetic fieldaligned circularly polarized EMIC waves and ponderomotively driven ion-acoustic perturbations. Within the framework of the two-fluid model, we obtain a time-dependent nonlinear Schrödinger equation (NLSE) coupled with the driven ion-acoustic density evolution equation. The pair of equations admits the existence of dark and grey envelope soliton solutions. Explicit profiles for these solutions are presented, and the relevance of our investigation to space plasmas will be discussed. It should be stressed that our results are different from those of Champeaux et al. (1999), who investigated the amplitude modulation of dispersive Alfvén waves based on a derivative NLSE deduced from the Hallmagnetohydrodynamic equations.

\section{The model}

We consider a uniform collisionless plasma consisting of ions (denoted by $i$; mass $m_{i}$, charge $q_{i}=+e ; e$ denotes the absolute value of the electron charge) and electrons (mass $m_{e}$, charge $q_{e}=-e$ ), which is embedded in an external magnetic field $\mathbf{B}=B_{0} \hat{z}$, where $B_{0}$ is the strength of the magnetic field and $\hat{z}$ is the unit vector along the z-axis. $n_{i / e, 0}$ will denote the ion/electron number density at equilibrium, where overall charge neutrality is assumed. In such a plasma, we are interested in investigating the nonlinear coupling between the lefthand circularly polarized EMIC waves having the electric field $\mathbf{F}=F(\hat{x}-i \hat{y}) \exp (i k z-i \omega t)+$ c.c. (complex conjugate), where $\mathbf{F} / 2=\left(E_{x}, E_{y}, 0\right)$, and low-phase-velocity (compared to the ion/electron thermal speeds $\left.v_{t h, i / e}=\left(T_{e} / m_{i / e}\right)^{1 / 2}\right)$ ion-acoustic (IA) perturbations, propagating in our mag- netized plasma. We will adopt a cold plasma model for the EMIC waves, assuming that $\left|\omega-\omega_{c, i / e}\right| \gg k v_{t h, i / e}$, where $\omega_{c, i / e}=q_{i / e} B_{0} / m_{i / e} c$ denotes the ion/electron cyclotron (gyro-) frequency. We also define the electron/ion plasma frequency $\omega_{p, e / i}=\left(4 \pi n_{i / e, 0} q_{i / e}^{2} / m_{e / i}\right)^{1 / 2}$, which is much smaller than the frequency of interest $\omega \gg \omega_{p, e / i}$. The vector(s) $\hat{x}(\hat{y})$ denote(s) the unit vector(s) along the $\hat{x}(\hat{y})$ axes, respectively.

For $\omega \lesssim \omega_{c, i} \ll\left|\omega_{c, e}\right|$, the frequency $\omega$ and the wave number $\mathbf{k}=k \hat{z}$ ( $k=2 \pi \lambda$, where $\lambda$ is the EMIC wave length) are related by the dispersion relation (Stix, 1992)

$k^{2} v_{A}^{2}=\frac{\omega^{2} \omega_{c, i}}{\omega_{c, i}-\omega}$,

where $v_{A}$ denotes the Alfvén velocity $v_{A}=B_{0} / \sqrt{4 \pi m_{i} n_{i, 0}}=c \omega_{c, i} / \omega_{p, i}$.

The nonlinear interaction between EMIC waves and ion acoustic (IA) perturbations produces an electric field envelope, which obeys (Karpman and Washimi, 1976, 1977; Shukla and Stenflo, 1984, 1985)

$i\left(\frac{\partial F}{\partial t}+v_{g} \frac{\partial F}{\partial z}\right)+P \frac{\partial^{2} F}{\partial z^{2}}-\Delta F=0$.

The group velocity $v_{g}=\omega^{\prime}(k)$ of the EMIC waves is

$v_{g}=\frac{2 k v_{A}^{2}}{\omega} \frac{\left(\omega-\omega_{c, i}\right)^{2}}{\omega_{c, i}\left(2 \omega_{c, i}-\omega\right)}$.

The group velocity dispersion coefficient $P=\omega^{\prime \prime}(k) / 2$ reads

$P=\left[1-\frac{v_{g}^{2}}{v_{A}^{2}} \frac{\omega_{c, i}^{3}}{\left(\omega_{c, i}-\omega\right)^{3}}\right] \frac{v_{g}}{2 k}$.

Combining Eq. (1) and Eq. (3) into Eq. (4), one may readily check that $P<0$ for all values of the wavenumber $k$.

The nonlinear frequency shift $\Delta$ arises due to the nonlinear interaction between the EMIC waves and the IA perturbations. It is given by

$$
\begin{aligned}
\Delta & =\frac{v_{g}}{2 k c^{2}} \sum_{j=e, i} \frac{\omega \omega_{p, j}^{2}}{\omega-\omega_{c, j}}\left[N+\frac{k \omega_{c, j}}{\omega\left(\omega-\omega_{c, j}\right)} v_{j, z}\right] \\
& =-\frac{k v_{g}}{2} N-k \frac{\partial}{\partial t}\left(\frac{\partial}{\partial z}\right)^{-1} N,
\end{aligned}
$$

where $N=\delta n_{e} / n_{e, 0}=\delta n_{i} / n_{i, 0}$ denotes the electron/ion density perturbation $\delta n_{e / i}$ scaled over the equilibrium density $n_{i, 0} / n_{i, 0}$. In deriving Eq. (6), we have used the fact that $v_{e, z} \approx v_{i, z} \approx-\partial_{t} \partial_{z}^{-1} N$.

The dynamics of the low-frequency IA perturbations is governed by the ion continuity equation

$\frac{\partial N}{\partial t}+\frac{\partial v_{i, z}}{\partial z}=0$,

and the ion momentum equation

$\frac{\partial v_{i, z}}{\partial t}+c_{s}^{2} \frac{\partial N}{\partial z}=\mathbf{f}$ 
where we have defined the sound velocity $c_{s}=$ $\left[\left(T_{e}+\gamma_{i} T_{i}\right) / m_{i}\right]^{1 / 2} \equiv\left[T_{0} / m_{i}\right]^{1 / 2}$. Here, $\gamma_{i}$ is the ion adiabatic index. The force appearing in the right-hand side of Eq. (8) is the ponderomotive force associated with the EMIC waves. It reads $\mathbf{f}=f \hat{z}$, where

$f=\frac{q_{i}^{2}}{m_{i}^{2} \omega_{c i}\left(\omega_{c i}-\omega\right)}\left(\frac{\partial}{\partial z}+\frac{2}{v_{g}} \frac{\partial}{\partial t}\right)|F|^{2}$.

Combining Eqs. (7) and (8) we obtain for the density perturbation

$$
\begin{aligned}
& \frac{\partial^{2} N}{\partial t^{2}}-c_{s}^{2} \frac{\partial^{2} N}{\partial z^{2}}= \\
& -\frac{q_{i}^{2}}{m_{i}^{2} \omega_{c i}\left(\omega_{c i}-\omega\right)}\left(\frac{\partial^{2}}{\partial z^{2}}+\frac{2}{v_{g}} \frac{\partial^{2}}{\partial t \partial z}\right)|F|^{2} .
\end{aligned}
$$

Equations (2) and (10), combined via Eq. (6), form a closed system, which describe the simultaneous evolution of the density perturbation $N$ and the electric field amplitude $F$. One notices in the right-hand side of Eq. (10) the ponderomotive effect $\left(\sim|F|^{2}\right)$ which acts on the plasma slow motion.

\section{Stationary EMIC envelopes}

Since we are looking into the (slow) dynamics of the electric field envelope, we may move to a reference system which moves at the group velocity $v_{g}$. Thus, we define the moving coordinates $z^{\prime}=z-v_{g} t$ and $t^{\prime}=t$. The Galilean variable transformation implies $\partial / \partial x \rightarrow \partial / \partial z^{\prime}$ and $\partial / \partial t \rightarrow \partial / \partial t^{\prime}-v_{g} \partial / \partial z^{\prime}$. Furthermore, in order to gain some analytical insight in the dynamics, we may assume a very slow time variation (i.e. $\left.\partial^{2} / \partial t^{\prime 2}, \partial^{2} / \partial z^{\prime} \partial t^{\prime} \ll \partial^{2} / \partial z^{\prime 2}\right)$. Equation (10) then becomes

$\frac{\partial^{2} N}{\partial z^{\prime 2}} \approx \frac{q_{i}^{2}}{m_{i}^{2} \omega_{c i}\left(\omega_{c i}-\omega\right)} \frac{1}{v_{g}^{2}-c_{s}^{2}} \frac{\partial^{2}}{\partial z^{\prime 2}}|F|^{2}$,

which, assuming a vanishing density perturbation at infinity (i.e. a localized excitation profile), can be integrated twice to yield

$N \approx \frac{q_{i}^{2}}{m_{i}^{2} \omega_{c i}\left(\omega_{c i}-\omega\right)} \frac{1}{v_{g}^{2}-c_{s}^{2}}\left(|F|^{2}-\left|F_{\infty}\right|^{2}\right)$,

where the integration constant $F_{\infty}$ denotes the (constant) field amplitude at infinity. Now, combining with Eq. (6), one obtains

$$
\begin{aligned}
\Delta & =\frac{k v_{g}}{2} \frac{q_{i}^{2}}{m_{i}^{2} \omega_{c i}\left(\omega_{c i}-\omega\right)} \frac{1}{v_{g}^{2}-c_{s}^{2}}\left(|F|^{2}-\left|F_{\infty}\right|^{2}\right) \\
& \equiv-Q\left(|F|^{2}-\left|F_{\infty}\right|^{2}\right) .
\end{aligned}
$$

The quantity $Q$, whose definition in obvious, determines the ponderomotive nonlinear effect on the evolution of the electric field $F$.

Equation (2), in combination with Eq. (13), may now be cast in the reduced form of a nonlinear Schrödinger equation (NLSE)

$i \frac{\partial \mathcal{E}}{\partial \tau}+p \frac{\partial^{2} \mathcal{E}}{\partial s^{2}}+q|\mathcal{E}|^{2} \mathcal{E}=0$, where $\mathcal{E}=F / \sqrt{4 \pi n_{i, 0} T_{0}}$ denotes the (reduced) electric field amplitude, and time and space have been scaled over the ion plasma period (inverse frequency) $\omega_{p, i}^{-1}$ and Debye length $\lambda_{D}=c_{s} / \omega_{p, i}$, respectively. The dimensionless space and time variables are $s=z^{\prime} / \lambda_{D}=z / \lambda_{D}-\left(v_{g} / \lambda_{D}\right) \tau$ (the sound velocity $\lambda_{D}$ was defined above) and $\tau=\omega_{p, i} t$.

The constant contribution to the right-hand side of Eq. (12) (related to the value of $F$ at infinity) was omitted in Eq. (14), since it simply leads to a linear phase shift in $\mathcal{E}$ : it is eliminated upon setting $\mathcal{E} \rightarrow \mathcal{E} \exp \left(i q\left|\mathcal{E}_{\infty}\right|^{2} \tau\right)$.

Equations (12) and (14) now determine the evolution profile of the relative density variation $N$ and the (reduced) field variation $\mathcal{E}$ (both quantities are dimensionless).

The analytical form of the (dimensionless) coefficients in Eq. (14) plays a crucial role in the description of the electromagnetic IC wave stability profile. The nonlinearity coefficient $q=Q 4 \pi n_{i, 0} T_{0} / \omega_{p, i}$ in Eq. (14) reads

$q=-\frac{k v_{g}}{2} \frac{q_{i}^{2}}{m_{i}^{2} \omega_{c i}\left(\omega_{c i}-\omega\right)} \frac{1}{v_{g}^{2}-c_{s}^{2}} \frac{4 \pi n_{i, 0} T_{0}}{\omega_{p, i}}$,

while the dispersion coefficient $p=P / \lambda_{D}^{2} \omega_{p, i}$ is now given by

$p=\frac{1}{\lambda_{D}^{2} \omega_{p, i}}\left[1-\frac{v_{g}^{2}}{v_{A}^{2}} \frac{\omega_{c, i}^{3}}{\left(\omega_{c, i}-\omega\right)^{3}}\right] \frac{v_{g}}{2 k}$.

\subsection{Reduced coefficients}

Combining the above definitions, the expressions for the coefficients $p$ and $q$ may be cast in a more physically transparent (and elegant) form, in terms of the reduced wavenumber $\tilde{k}=c k / \omega_{p, i}=k v_{A} / \omega_{c, i}$. One thus obtains the final (reduced) expression, for the dispersion coefficient in Eq. (14)

$p=-p_{0}\left[1-\frac{\tilde{k}\left(6+\tilde{k}^{2}\right)}{\left(4+\tilde{k}^{2}\right)^{3 / 2}}\right]$,

where $\quad p_{0}=-p(\tilde{k}=0)=\frac{v_{A}^{2}}{2 c_{s}^{2}} \frac{\omega_{p, i}}{\omega_{c, i}}=\frac{c^{2}}{2 c_{s}^{2}} \frac{\omega_{c, i}}{\omega_{p, i}}=\frac{c}{c_{s}} \frac{1}{\sqrt{2 \beta}}$ tice the appearance of the plasma-beta parameter $\beta=2 c_{s}^{2} / v_{A}^{2}=8 \pi n_{i, 0} T_{0} / B_{0}^{2}$ in all forthcoming formulae). Retain that the quantity within brackets turns out to be positive, for every value of the wavenumber $\tilde{k}$, so $p$ is negative.

The nonlinearity coefficient in Eq. (14) becomes

$q=\frac{\omega_{p, i}}{\omega_{c, i}} \frac{1}{1-\frac{v_{g}^{2}}{c_{s}^{2}}} \frac{\tilde{k}}{\left(4+\tilde{k}^{2}\right)^{1 / 2}}$.

Note that $q$ is positive (negative) depending on whether the group velocity is subsonic (supersonic, respectively), i.e. for wavenumbers higher (lower) than a critical wavenumber $k_{c r}$ (cf. the detailed discussion in Sect. 6). Upon substitution from Eq. (3) into Eq. (18), one obtains the alternative expression

$q(\tilde{k} ; \beta)=\frac{c}{c_{s}} \sqrt{\frac{\beta}{2}}\left(1-\frac{\beta_{c r}(\tilde{k})}{\beta}\right)^{-1} \frac{\tilde{k}}{\left(4+\tilde{k}^{2}\right)^{1 / 2}}$, 
where $\beta_{c r}(\tilde{k}) \equiv \beta v_{g}^{2} / c_{s}^{2}$ is explicitly evaluated in Sect. 6; see Eq. (30).

We also define the coefficient ratio

$\eta \equiv \frac{p}{q}=\frac{1}{\beta} \frac{6 \tilde{k}+\tilde{k}^{3}-\left(4+\tilde{k}^{2}\right)^{3 / 2}}{\tilde{k}\left(4+\tilde{k}^{2}\right)^{1 / 2}}\left(1-\frac{v_{g}^{2}}{c_{s}^{2}}\right)$,

for later reference. We observe that $\eta$ is negative (positive) in the subsonic (supersonic, respectively) envelope case; see Fig. 5; cf. Sect. 6.

The density perturbation $N$ is given by Eq. (12), which now takes the form

$$
\begin{aligned}
N & =\frac{\omega_{p, i}^{2}}{\omega_{c, i}\left(\omega_{c, i}-\omega\right)} \frac{c_{s}^{2}}{v_{g}^{2}-c_{s}^{2}}\left(|\mathcal{E}|^{2}-\left|\mathcal{E}_{\infty}\right|^{2}\right) \\
& \equiv \frac{\omega_{p, i}^{2}}{\omega_{c, i}^{2}} \frac{c_{s}^{2}}{v_{g}^{2}-c_{s}^{2}} \frac{2}{2+\tilde{k}\left(\tilde{k}-\sqrt{4+\tilde{k}^{2}}\right)}\left(|\mathcal{E}|^{2}-\left|\mathcal{E}_{\infty}\right|^{2}\right) \\
& \equiv \alpha\left(|\mathcal{E}|^{2}-\left|\mathcal{E}_{\infty}\right|^{2}\right) .
\end{aligned}
$$

Note that $N$ scales as $\beta^{-1} \sim B^{-2}$, (for low magnetic field values, viz. $\left.\omega_{c, i} \ll \omega_{p, i}\right)$, implying that for a lower (higher) $\beta$ value - i.e. for a higher (lower) magnetic field value - the density variation will be less (more, respectively) important, for a given electric field $E$. As for the pre-factor $\alpha$ (an analytic function of $\tilde{k}$ ), it takes positive (negative) values for $v_{g}$ above (below) $c_{s}$; recall that $\omega \lesssim \omega_{c, i}$ here).

The evolution Eqs. (14) and (21) for the (reduced) electric field $\mathcal{E}$ and density $N$, combined with the expressions (17)(20) (in addition to Eqs. 1 and 3 above), model the simultaneous propagation of the localized field and density perturbations we aim at modeling. These equations form the basis of the analysis which follows.

\section{Modulational stability analysis}

According to the generic NLS formalism, exposed in detail elsewhere (see e.g. Hasegawa, 1975; Shukla and Stenflo, 1986; Remoissenet, 1994; Sulem and Sulem, 1999), one expects the field envelope $\mathcal{E}$, which is associated with the EMIC waves, to be modulationally stable (unstable), if the product $p q$ is negative (positive), i.e. for a group velocity $v_{g}$ below (above) the sound velocity $c_{s}$, here. To see this, one may first check that the NLSE is satisfied by the plane wave solution $\mathcal{E}(s, \tau)=\mathcal{E}_{0} e^{i q\left|\mathcal{E}_{0}\right|^{2} T}+$ c.c. The standard (linear) stability analysis then shows that a linear modulation with the frequency $\Omega$ and the wavenumber $\kappa$ obeys the dispersion relation

$\Omega^{2}(\kappa)=p \kappa^{2}\left(p \kappa^{2}-2 q\left|\mathcal{E}_{0}\right|^{2}\right)$

whose right-hand side is positive if $p q<0$, viz. $\Omega$ is real. Otherwise, for $p q>0$, a purely growing instability occurs for $\kappa \leq \kappa_{c r}=(q / p)^{1 / 2}\left|\mathcal{E}_{0}\right|$. The growth rate $\sigma=\operatorname{Im}(\Omega)$ attains a maximum value $\sigma_{\max }=q\left|\mathcal{E}_{0}\right|^{2}$ (Shukla and Stenflo, 1986).

\section{Envelope excitations}

It is known from the existing theory (Hasegawa, 1975; Remoissenet, 1994) that the NLSE (Eq. 14) is an integrable dynamical system which admits, among others, localized solutions in the form of envelope solitons of the bright $(p q>0)$ or dark $(p q<0)$ type. Analytical expressions for these solutions are found by inserting the trial function $\mathcal{E}=\mathcal{E}_{0} \exp (i \Theta)$ in Eq. (14) and then separating real and imaginary parts in order to determine the (real) functions $\mathcal{E}_{0}(s, \tau)$ and $\Theta(s, \tau)$. Details on the derivation of their analytic form can be found e.g. in (Fedele and Schamel, 2002), so only the final expressions for dark envelope solitons (which are of interest to us here) will be given in the following. Let us retain that this ansatz amounts to a total electric field whose components $E_{j}(z, t)(j=x, y)$ are essentially equal to

$E_{j}(z, t)=2 \mathcal{E}_{0} \cos (k z-\omega t+\Theta)$,

where the localized field envelope amplitude $\mathcal{E}_{0}$ and the (small) phase shift $\Theta$ will be determined, case by case. The (co-propagating) density perturbation $N$ is then readily given by Eq. (21). These bi-solitons, which represent the joint propagation of $\mathcal{E}$ and $N$, may be of different types, which are outlined in the following (with respect to our case of interest).

\subsection{Bright-type EMIC envelope excitations}

In the modulationally unstable case $(\eta=p / q>0)$, i.e. for $v_{g}>c_{s}$ (supersonic case), modulated EMIC wave collapse may result in the formation of a localized pulse-shaped slowly-varying envelope (a soliton solution of the NLSE, 14), which modulates the carrier wave (see Figs. 1 and 2). This bright type (pulse) envelope solution (with $E_{\infty}=0$ ) is given by the expression (Fedele and Schamel, 2002)

$\begin{aligned} \mathcal{E}_{0} & =\hat{\mathcal{E}}_{0} \operatorname{sech}\left(\frac{s-v_{e} \tau}{L}\right), \\ \Theta & =\frac{1}{2 p}\left[v_{e} s+\left(\Omega-\frac{v_{e}^{2}}{2}\right) \tau\right],\end{aligned}$

where $v_{e}$ is the envelope velocity; $L$ and $\Omega$ represent the pulses spatial width and oscillation frequency (at rest), respectively. In our problem, the bright-type localized envelope solutions may occur and propagate in the plasma if a sufficiently long wavelength is chosen, so that the product $p q$ is positive. We note that the pulse width $L$ and the maximum amplitude $\hat{\mathcal{E}}_{0}$ satisfy $L \hat{\mathcal{E}}_{0}=(2 p / q)^{1 / 2}=(2 \eta)^{1 / 2}=$ constant.

In this (supersonic) case, the density field variation $N=\delta n / n_{0}=\alpha\left(|\mathcal{E}|^{2}-\left|\mathcal{E}_{\infty}\right|^{2}\right)$ (recall Eq. 21) is given by

$N=\alpha \hat{\mathcal{E}}_{0}^{2} \operatorname{sech}^{2}\left(\frac{s-v_{e} \tau}{L}\right)>0$,

where $L=(2|\eta|)^{1 / 2} / \hat{\mathcal{E}}_{0}$. We observe that the density variation will be positive in this case, since $\alpha>0$ for $v_{g}>c_{s}$ (and $\mathcal{E}_{\infty}=0$ here); recall that $\alpha$ was defined in Eq. (21) above. Bright 


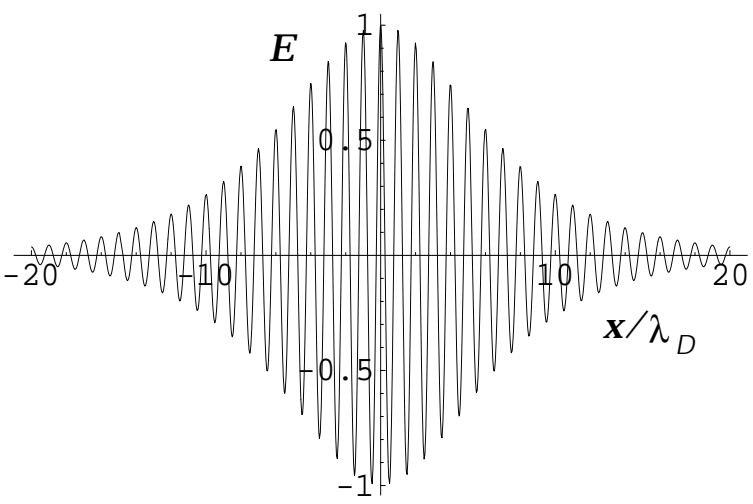

(a)

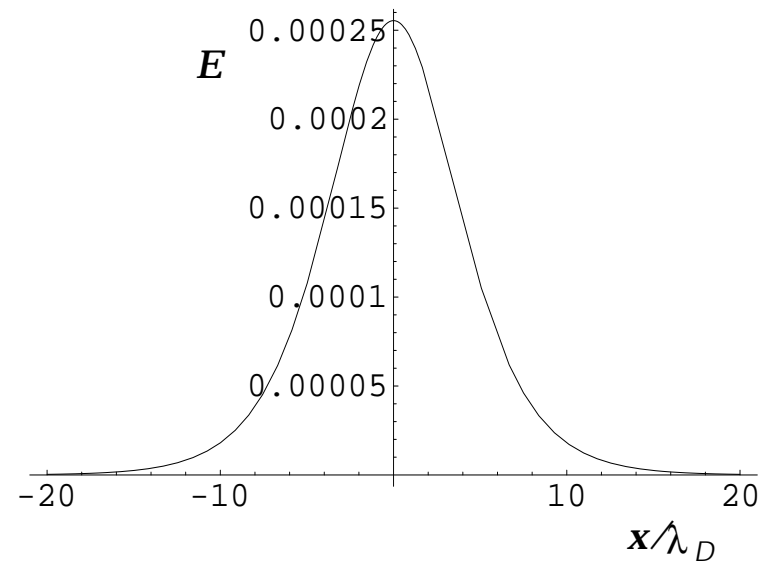

(b)

Fig. 1. Bright type (pulse) soliton solution of the NLS Eq. (14): (a) the field waveform $\mathcal{E}$ (scaled by its maximum value $\hat{\mathcal{E}}_{0}$ ) vs. the (reduced) position variable $x / \lambda$, as given by Eqs. (23) and (24); (b) the corresponding (normalized) density variation $\delta n / n_{0}=N$ (scaled by $\frac{c^{2}}{c_{s}^{2}} \hat{\mathcal{E}}_{0}^{2}$ ), as given by Eq. (25). The indicative parameter values in this plot are: $\beta=0.02, L=5, \tilde{k}=c k / \omega_{p, i}=0.3$ and $v_{e}=0$.

electric field envelope excitations will therefore be associated with a positive density variation, i.e. a co-propagating localized hump (pulse) in a constant (elsewhere) density profile.

It may be stressed that the finite amplitude localized envelope excitations presented here are intrinsically different in form (and obey different physics) from the pulse-like smallamplitude localized structures (solitons) typically found via the Korteweg-de Vries (KdV) theory, although a link has been attempted between the two theories; see in (Fedele, 2002) for a critical discussion; also in (Grimshaw, 2005) for an interesting recent investigation, with respect to the $\mathrm{mKdV}$ equation. Also recall that those localized pulse excitations are characterized by a spatial width $L$ and a maximum amplitude $\hat{\mathcal{E}}_{0}$ which satisfy $\hat{\mathcal{E}}_{0} L^{2}=$ constant, unlike the envelope structures above, which satisfy $\hat{\mathcal{E}}_{0} L=$ constant $(\sim \sqrt{|\eta|}$, see above) instead. This property of envelope excitations may serve as a distinguishing signature, in order to identify them e.g. in space observation data.

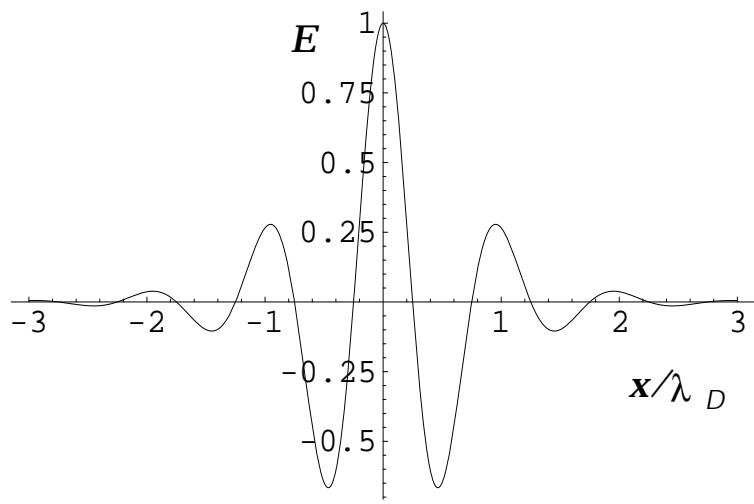

(a)

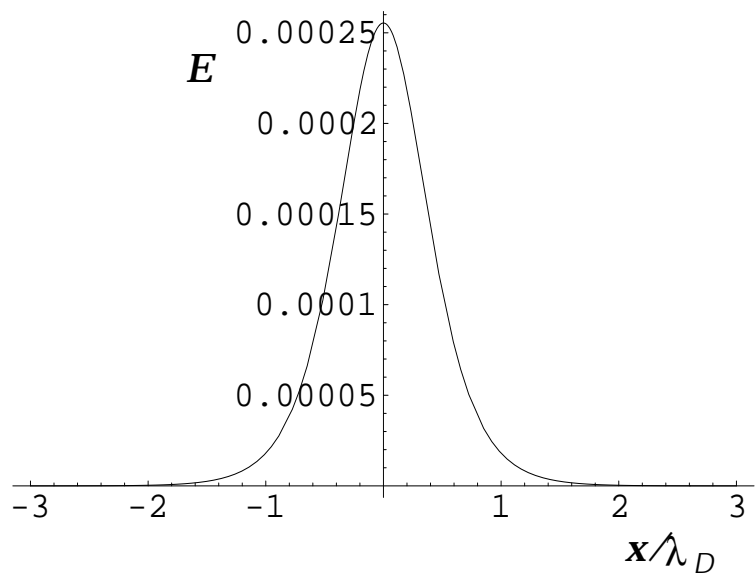

(b)

Fig. 2. Bright type (pulse) soliton solution of the NLS Eq. (14): (a) the field waveform $\mathcal{E}$ (scaled by its maximum value $\hat{\mathcal{E}}_{0}$ ) vs. the (reduced) position variable $x / \lambda$, as given by Eqs. (23) and (24); (b) the corresponding (normalized) density variation $\delta n / n_{0}=N$ (scaled by $\frac{c^{2}}{c_{s}^{2}} \hat{\mathcal{E}}_{0}^{2}$ ), as given by Eq. (25). Same parameter values as in Fig. 1, except $a(n)$ (extremely low) choice of $L=0.5$.

\subsection{Dark-type EMIC envelope excitations}

If $\eta=p / q<0$, i.e. for $v_{g}<c_{s}$ (subsonic case), modulated EMIC waves can propagate in the form of black envelope solitons (holes) (Fedele and Schamel, 2002) (see Fig. 3)

$$
\begin{aligned}
\mathcal{E}_{0} & =\frac{\sqrt{2|\eta|}}{L}\left|\tanh \left(\frac{s-v_{e} \tau}{L}\right)\right|, \\
\Theta & =\frac{1}{2 p}\left[v_{e} s+\left(2 p q \hat{\mathcal{E}}_{0}^{2}-\frac{v_{e}^{2}}{2}\right) \tau\right],
\end{aligned}
$$

which represents a localized region of reduced wave density (avoid). Note that the excitation width $L^{\prime}$ is inversely proportional to the amplitude $\hat{\mathcal{E}}_{0}=(2|\eta|)^{1 / 2} / L$.

Modulated EMIC wave packets may also propagate as grey envelope solitons (Fedele and Schamel, 2002), of the form (see Fig. 4)

$$
\mathcal{E}_{0}=\frac{\sqrt{2|\eta|}}{|d| L^{\prime}}\left\{1-d^{2} \operatorname{sech}^{2}\left(\frac{s-v_{e} \tau}{L^{\prime}}\right)\right\}^{1 / 2},
$$




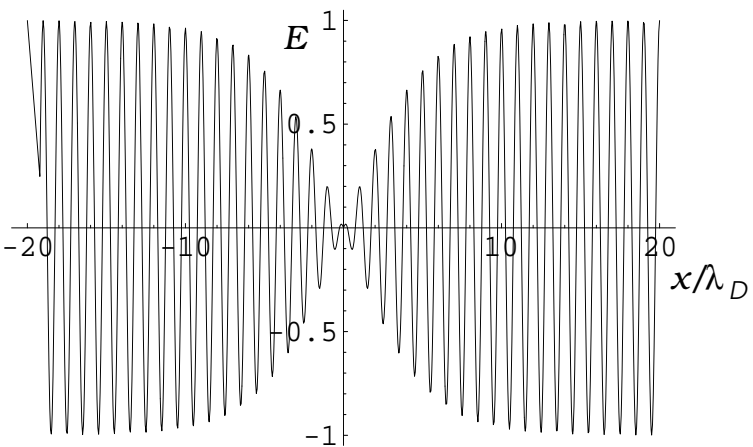

(a)

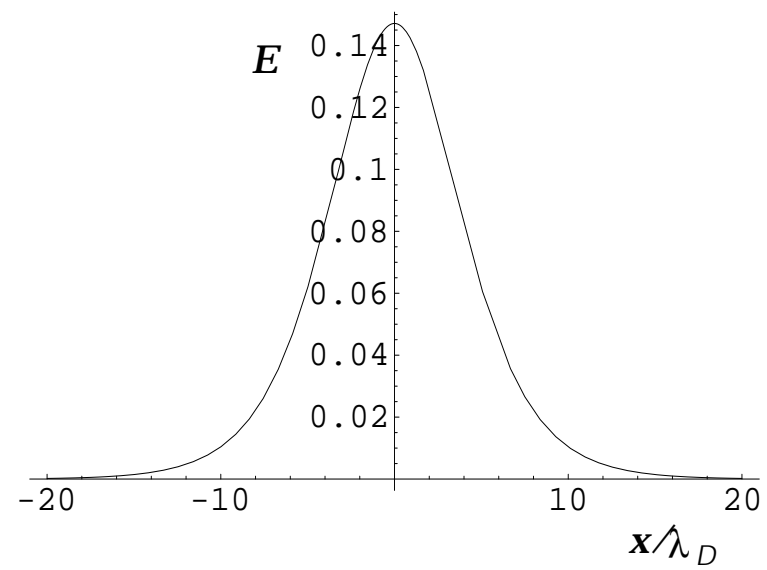

(b)

Fig. 3. Black type (hole) soliton solution of the NLS Eq. (14): (a) the field waveform $\mathcal{E}$ (scaled by its maximum value $\hat{\mathcal{E}}_{0}$ ) vs. the (reduced) position variable $x / \lambda$, as given by Eq. (23) and (26); (b) the corresponding (normalized) density variation $\delta n / n_{0}=N$ (scaled by $\left.\frac{c^{2}}{c_{s}^{2}} \hat{\mathcal{E}}_{0}^{2}\right)$, as given by Eq. (29). The indicative parameter values in this plot are: $\beta=0.02, L=5, \tilde{k}=c k / \omega_{p, i}=3$ and $v_{e}=0$.

where the nonlinear phase correction $\Theta=\Theta(X, T)$ now bears the complex expression

$$
\begin{array}{r}
\Theta=\frac{1}{2 p}\left[V_{0} s-\left(\frac{1}{2} V_{0}^{2}-2 p q \hat{\mathcal{E}}_{0}^{2}\right) \tau+\Theta_{0}\right] \\
-a \sin ^{-1} \frac{d \tanh \left(\frac{s-v_{e} \tau}{L^{\prime}}\right)}{\left[1-d^{2} \operatorname{sech}^{2}\left(\frac{s-v_{e} \tau}{L^{\prime}}\right)\right]^{1 / 2}},
\end{array}
$$

(Fedele and Schamel, 2002). Here, $\Theta_{0}$ is a constant phase; $a$ (=土1) denotes the product $\operatorname{sign} a=\operatorname{sign}(p) \times \operatorname{sign}\left(v_{e}-V_{0}\right)$. Again, the pulse width $L^{\prime}=(2|\eta|)^{1 / 2} /\left(|d| \hat{\mathcal{E}}_{0}\right)$ is inversely proportional to the amplitude $\hat{\mathcal{E}}_{0}$, and now also depends on an independent real parameter $d$, which regulates the modulation depth; $d$ is given by: $d^{2}=1+\left(v_{e}-V_{0}\right)^{2} /\left(2 p q \hat{\mathcal{E}}_{0}^{2}\right) \leq 1 . \quad V_{0}$ is an independent real constant which satisfies (see details in Fedele and Schamel, 2002): $\quad V_{0}-\sqrt{2|p q| \hat{\mathcal{E}}_{0}^{2}} \leq v_{e} \leq V_{0}+\sqrt{2|p q| \hat{\mathcal{E}}_{0}^{2}}$. This localized excitation represents a localized region of negative wave density (a propagating void), with a finite (non zero) amplitude

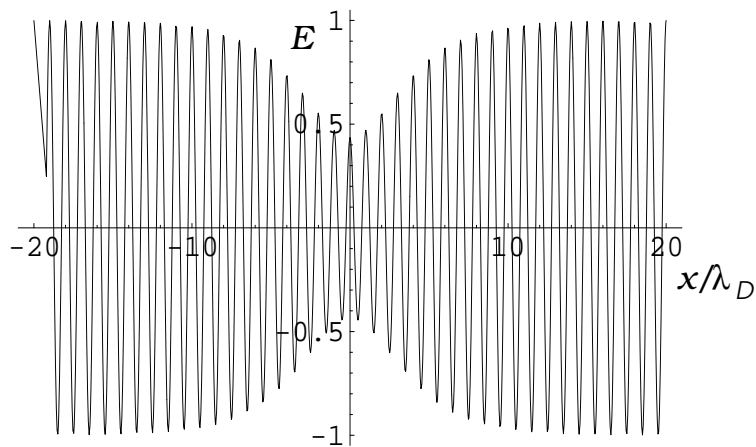

(a)

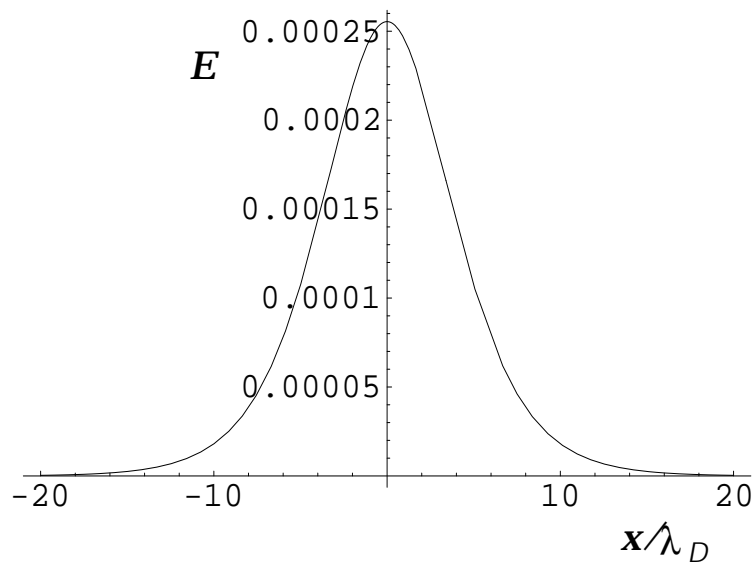

(b)

Fig. 4. Grey type soliton solution of the NLS Eq. (14): (a) the field waveform $\mathcal{E}$ (scaled by $\frac{c^{2}}{c_{s}^{2}} \hat{\mathcal{E}}_{0}^{2}$ ) vs. the (reduced) position variable $x / \lambda$, as given by Eqs. (23), (27) and (28); (b) the corresponding (normalized) density variation $\delta n / n_{0}=N$ (scaled by $\frac{c}{c_{s}} \hat{\mathcal{E}}_{0}^{2}$ ), as given by Eq. (29). The indicative parameter values in this plot are: $\beta=0.02, L=5, \tilde{k}=c k / \omega_{p, i}=3, d=0.9$ and $v_{e}=0$. Notice that the field amplitude never reaches zero (cf. Fig. 3).

at $s=0$. For $|d|=1$ (thus $V_{0}=v_{e}$ ), one recovers the dark envelope soliton presented above, which is characterized by a vanishing field amplitude (and density perturbation) at $s=0$.

In both black- and grey-type cases, the density field variation $N=\delta n / n_{0}=\alpha\left(|\mathcal{E}|^{2}-\left|\mathcal{E}_{\infty}\right|^{2}\right)$ (recall Eq. 21) is given by

$N=-\alpha \hat{\mathcal{E}}_{0}^{2} \operatorname{sech}^{2}\left(\frac{s-v_{e} \tau}{L}\right)<0$,

where $L=(2|\eta|)^{1 / 2} / \hat{\mathcal{E}}_{0}$ (see that the result does not depend on the parameter $d$ ). We observe that the density variation will be positive in this case (as well as in the bright case, above), since $\alpha<0$ for $v_{g}<c_{s}$ (and $|\mathcal{E}|<\left|\mathcal{E}_{\infty}\right|$ here); recall that $\alpha$ was defined in Eq. (21) above. Dark-type (either black- or grey) electric field envelope excitations will therefore be associated with a positive density variation, i.e. a co-propagating localized pulse (hump) in a constant (elsewhere) density profile. 


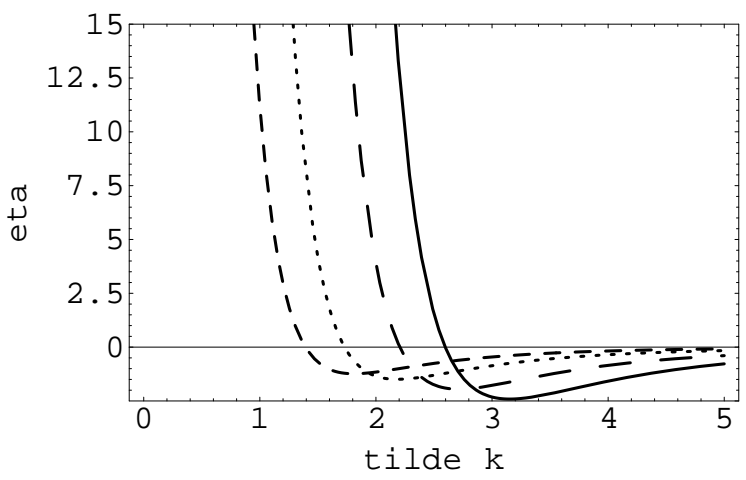

Fig. 5. The function $\eta=p / q$ (cf. Eq. 20), is depicted vs. the reduced wavenumber $\tilde{k}=k c / \omega_{p, i}$. Recall that this quantity determines the bi-soliton width, viz. $L \sim \sqrt{|\eta|}$ (for given magnetic field $B_{0}$ and electric field maximum intensity $E_{0}$ ). Four different values of the plasma $\beta$ parameter were taken here: 0.01 (solid line); 0.02 (long-dashed line)); 0.05 (dotted line); 0.1 (short-dashed line). See that $\eta$ changes sign at a critical wave number value, which depends on $\beta$ (and so does, in fact, $\alpha$; cf. Fig. 6 below).

\section{Envelope soliton characteristics: Sub- vs. Supersonic excitations}

Recall that the quantities $\eta$ and $\alpha$ (defined in Eqs. 20 and 21) determine the magnitude of the field envelope and the associated density perturbation, respectively. In specific, for a given electric field intensity $\mathcal{E}$, the spatial extension (width) of the localized field variation (field envelope excitation) varies as $L \propto|\eta|^{1 / 2} / \hat{\mathcal{E}}_{0}$. Stronger/weaker electric field amplitude values therefore correspond to narrower/wider (respectively) excitations (electric field holes, accompanied by density dips).

For a given value of $\hat{\mathcal{E}}_{0}$, the excitation width depends on the magnetic field as: $L \sim \sqrt{|\eta|} \sim \omega_{c, i} / \omega_{p, i} \sim B^{1}$, while the density variation depth $N_{0}=\alpha \hat{\mathcal{E}}_{0}^{2}$ scales as $\sim B^{-2}$. Stronger magnetic field (i.e. lower plasma $\beta$ ) values therefore correspond to wider field and density excitations, while the latter ones will be shorter (for higher $B$ ).

As far as the dependence on the wave number $k$ is concerned, in the positive $\eta$ (low $k$, supersonic bright EMIC envelope) region we find that $\eta$ and $\alpha$ bear a decreasing and increasing, respectively, behaviour, in terms of $k$ (see Figs. 5 and 6). Therefore, shorter wavelength $\lambda$ (i.e. higher wavenumber $k$ ) values correspond to narrower localized field bright localized envelope EMIC wave packets, which are accompanied by narrower and higher density humps (compressive pulses). On the other hand, in the negative $\eta$ (higher $k$, subsonic dark EMIC envelope) region we find that (the absolute value of) $\eta$ decreases with $k$, while (the absolute value of) $\alpha$ decreases between two $k$ values and then increases monotonically (see Figs. 5 and 6). Dark envelope solitons (holes) will therefore be narrower, for higher $k$, while the density compression region accompanying them will generally be higher, for larger $k$.

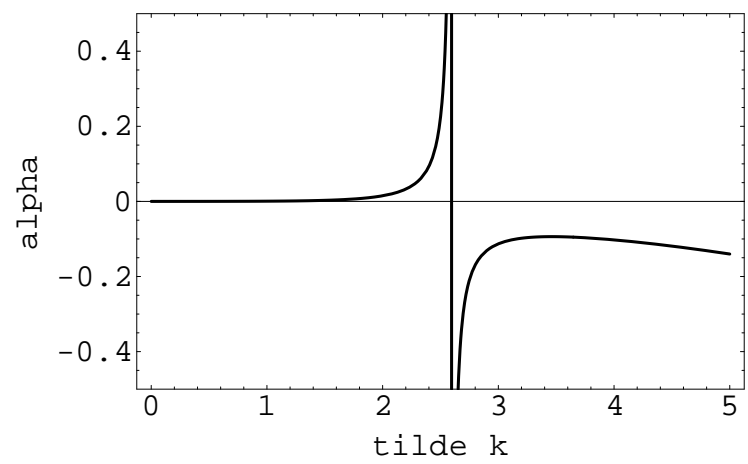

(a)

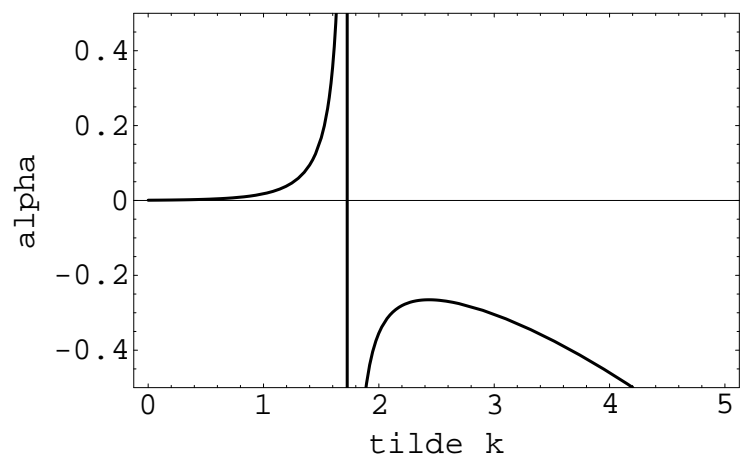

(b)

Fig. 6. The (dimensionless) function $\alpha$ (scaled by $c^{2} / c_{s}^{2}$ ) is depicted vs. the reduced wavenumber $\tilde{k}=k c / \omega_{p, i}$. Recall that this quantity determines the density variation height (for given magnetic field $B_{0}$ and electric field maximum intensity $E_{0}$ ). Two different values of the plasma $\beta$ parameter were taken here: (a) 0.01 ; (b) 0.05 . See that $a$ changes sign at a critical wave number value (cf. Fig. 5), near which it takes rather high values (near or above 1), implying important density variations in that wave number region.

We saw that the sign of the quantity $\eta=p / q$, which determines the type of EMIC envelope excitation which may occur in the plasma, depends on the ratio $v_{g} / c_{s}$. Combining the above definitions, we obtain the form

$\frac{v_{g}}{c_{s}}=\left(\frac{2}{\beta}\right)^{1 / 2}\left(\frac{2+\tilde{k}^{2}}{\sqrt{4+\tilde{k}^{2}}}-\tilde{k}\right) \equiv\left(\frac{\beta_{c r}}{\beta}\right)^{1 / 2}$.

We have defined the convenient quantity $\beta_{c r}$, in fact an exact function of $\tilde{k}=c k / \omega_{p, i}$, in order to formulate an explicit criterion for the relative magnitude of the ratio $v_{g} / c_{s}$ with respect to unity: for a given $\tilde{k}$, values of the plasma $\beta$ parameter above (below) the critical value $\beta_{c r}$ correspond to a subsonic (supersonic) EMIC envelope. Now, note that $\beta_{c r}$ is a monotonic (in fact descending) function of $\tilde{k}$ (see Fig. 7). Therefore, for a given value of $\beta$, the solution of the equation $\beta=\beta_{c r}$ with respect to $\tilde{k}$, say $k=k_{c r}$ (viz. $\beta_{c r}\left(k_{c r}\right)=\beta$ ), provides the value of the wavenumber $k_{c r}$ at which $\eta=p / q$ (or simply $q$, in fact) changes sign: wavenumber values above (below) $k_{c r}$ will correspond to subsonic (supersonic) EMIC wave packets, i.e. to dark (bright) type envelope excitations. Upon simple inspection of Fig. 7, we see that bright envelope 


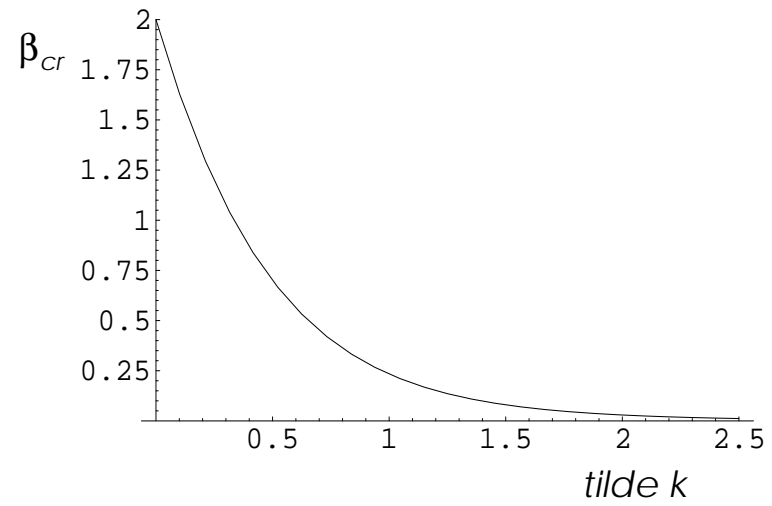

(a)

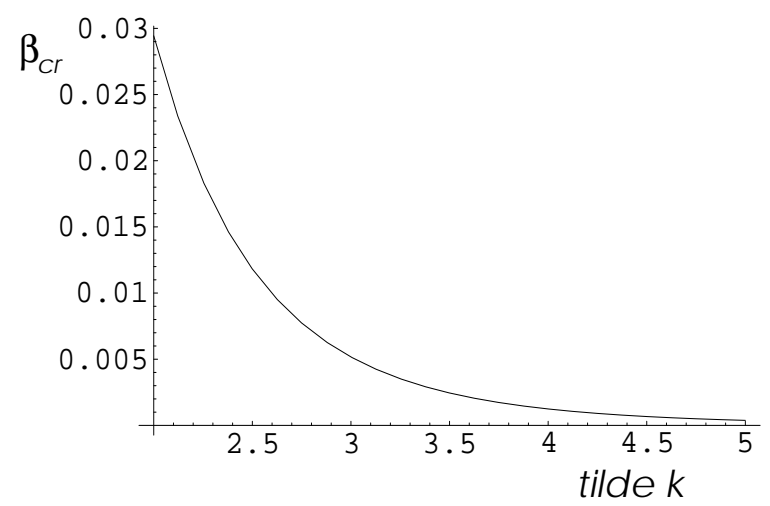

(b)

Fig. 7. The critical value $\beta_{c r}$ (defined in Eq. 30) is depicted vs. the reduced wavenumber $\tilde{k}=k c / \omega_{p, i}$, in two different wave number regions. Recall that the value of $\beta_{c r}$ (compared to the ambient plasma $\beta$ ) determines the sub-(super-) sonic character of localized EMIC wave packets: $\beta>\beta_{c r}$ implies $v_{g}<c_{s}$ (subsonic), and vice versa; therefore, for a given $\beta$ value, lower wave number $k$ (i.e. higher $\lambda$ ) values correspond to the supersonic envelope case, and vice versa.

solitons will be rather privileged; indeed, a typical (low) value of $\beta \approx 0.01$ provides a critical wave number $\tilde{k} \approx 2.5$, i.e. a critical wave length $\lambda_{c r} \approx 2 \pi / 2.5$ times the plasma skin depth $c / \omega_{p, i}$, suggesting that most (realistic) wavelength values correspond to supersonic (bright envelope) EMIC wave packets. However, the value of the plasma $\beta$ parameter depends on the plasma environment considered, and may not always be negligibly small; read the discussion in (Crary et al., 1998; Gary, 2001); for instance, exceptionally high $\beta$ values were reported by the Galileo mission to Jupiter, during its passing just outside the orbit of Io (Russell et al., 2001).

Summarising, a EMIC wave packet which is characterized by a wave length longer than a certain critical value $\lambda_{c r}$ (which depends on the plasma $\beta$ value; $\mathrm{cf}$. above) will tend to localize its energy by forming bright envelope solitons; this case may refer to the majority of space plasma EMIC waves, for a given realistic (very low) value of $\beta$. On the other hand, EMIC wave packets with wavelengths shorter $\lambda_{c r}$ will present a tendency to propagate as dark (black/grey) localized envelope forms (holes, voids).

\section{Conclusions}

We have studied the parametric interaction between large amplitude magnetic field-aligned, circularly polarized EMIC waves and ponderomotively driven non-resonant ionacoustic density perturbations in plasmas. By adapting and then solving the associated cubic nonlinear Schrödinger equation, we have shown that the EMIC waves are modulationally stable against quasi-stationary density perturbations, and that the modulated electric field wavepacket can appear in the form of either supersonic bright or subsonic dark (dark or grey) type solitary wave envelope solitons (exact solutions of the NLSE) for the EMIC wave electric field envelope. The former (bright-type field envelope solitons) represent propagating envelope pulses (modulating the electric field), while the latter (dark-type field envelope solitons) are reduced electric field intensity regions (modulating the electric field). Both types of localized excitations are associated with co-propagating localized positive density variations (humps).

Localized modulated wave packets are encountered in abundance during spacecraft observations (Cattell et al., 1998; McFadden et al., 1998; Stasiewicz et al., 2000; Chaston et al., 2002; Meziane et al., 2004a, b), and they are often associated with localized field and/or density variations simultaneously recorded (Pottelette et al., 1999; Alpert, 2001; Santolik, 2003). Our results may be of relevance to these observations.

Also, from a fundamental point of view, field modulation due to ponderomotive coupling to low-frequency turbulence is a generic mechanism (previously considered with respect to a variety of plasma modes), to be distinguished (despite their similar manifestation) from the amplitude (auto-) modulation due to carrier wave self-interactions, previously considered; see e.g. (Kourakis and Shukla, 2005) and references therein. Both theories account for moderately increased amplitude oscillations, to be distinguished from (and thought of as complementary to) weak-amplitude (e.g. KdV, mKdV) and arbitrary amplitude (e.g. Sagdeev) theories.

A satisfactory exact theory for the formation and dynamics of envelope localized plasma structures e.g. in the magnetosphere has always been lacking in the literature, so the present study aims in partially filling this gap.

Acknowledgements. I.K. is grateful to Gregor Morfill at MaxPlanck-Institut für extraterrestrische Physik (Garching, Germany) for the award of a fellowship (project: "Komplexe Plasmen"). Partial support from the Deutsche Forschungsgemeinschaft through Sonderforschungsbereich (SFB) 591 - Universelles Verhalten Gleichgewichtsferner Plasmen: Heizung, Transport und Strukturbildung is also gratefully acknowledged.

Edited by: R. Grimshaw

Reviewed by: two referees 


\section{References}

Alpert, Y.: Resonance nature of the magnetosphere, Physics Reports, 339, 323-444, 2001.

Alport, M. J., Barrett, P. J., and Behrens, M. A.: Selective destabilisation of ion cyclotron modes, Plasma Physics, 25, 1059-1064, 1983.

Alport, M. J. and van Niekerk, E.: The propagation of ion cyclotron waves in a Q-machine, Plasma Phys. Control. Fusion, 32, 637649, 1990.

Alport, M. J., Koepke, M. E., Sheridan, T. E., Amatucci, W. E., and Carrol III, J. J.: Asymmetric spectral broadening of modulated ion cyclotron waves, Geophys. Res. Lett., 21, 1011-1014, 1994.

Baumjohann, W. and Treumann, R. A.: Basic Plasma Space Physics, Imperial College Press, London, 1996.

Cattaert, T., Kourakis, I., and Shukla, P. K.: Envelope solitons associated with electromagnetic waves in a magnetized pair plasma, Physics of Plasmas, 12, 1, 012319/1-6, 2005.

Cattell, C., Bergmann, R., Sigsbee, K., Carlson, C. W., Chaston, C. C., Ergun, R. E., McFadden, J. P., Mozer, F. S.: The association of electrostatic ion cyclotron waves, ion and electron beams and field-aligned currents: FAST observations of an auroral zone crossing near midnight, Geophys. Res. Lett., 25, 12, 2053-2056, 1998

Champeaux S., Passot, T., and Sulem, P. L.: Dissipation of weakly dispersive Alfvén waves, Physics of Plasmas, 6, 1, 413-416, 1999.

Chaston, C. C., Bonnel, J. W., McFadden, J. P., Ergun, R. E., and Carlson, C. W.: Electromagnetic ion cyclotron waves at proton cyclotron harmonics, J. Geophys. Res., 107, A11, 1351/1-19, 2002

Cranmer, S. R., Field, G. B., and Kohl, J. L.: Spectroscopic constraints on models of ion-cyclotron resonance heating in the polar solar corona and high speed solar wind, Astrophys. J., 518, 937-947, 1999.

Crary, F. J., Bagenal, F., Frank, L. A., and Paterson, W. R.: Galileo plasma spectrometer measurements of composition and temperature in the Io plasma torus, J. Geophys. Res., 103, 29359-29370, 1998.

D'Angelo, N.: Plasma waves and instabilities in the polar cusp: A Review, Rev. Geophys. and Sp. Phys., 15, 299-307, 1977.

Eliasson, B. and Shukla, P. K.: Theoretical and numerical studies of density modulated whistlers, Geophys. Res. Lett., 31, 17, L17802/1-4, 2004.

Fedele, R. and Schamel, H.: Solitary waves in the Madelung's Fluid: A Connection between the nonlinear Schrödinger equation and the Korteweg-de Vries equation, Eur. Phys. J. B, 27, 313-320, 2002.

Fedele, R.: Envelope Solitons versus Solitons, Phys. Scripta, 65, 502-508, 2002

Gary, G. A.: Plasma beta above a solar active region: rethinking the paradigm, Solar Physics, 203, 71-86, 2001.

Gomberoff, L.: Ion Cyclotron Waves in Solar Plasmas, Brazilian Journal of Physics, 26, 2, 426-451, 1996.

Greene, G. J. and Gould, R. W.: Observations of ICRF Wave-packet Propagation in a Tokamak Plasma, Phys. Fluids B3, 406-413, 1991.

Grimshaw, R., Pelinovsky, E., Talipova, T., Ruderman, M., and Erdélyi, R: Short-Lived Large-Amplitude Pulses in the Nonlinear Long-Wave Model Described by the Modified Korteweg-De Vries Equation, SIAM Studies in Applied Mathematics, 114, 2, 189-210, 2005.
Guglielmi, A., Hayashi, K., Lundin, R, and Potapov, A.: Ponderomotive impacts of ion cyclotron waves on the ions in the equatorial zone of the magnetosphere, Earth Planets Space, 51, 1297 $1308,1999$.

Hasegawa, A.: Stimulated Modulational Instabilities of Plasma Waves, Phys. Rev. A, 1, 1746-1750, 1970.

Hasegawa, A.: Theory and Computer Experiment on Self-Trapping Instability of Plasma Cyclotron Waves, Phys. Fluids 15, 870 $881,1972$.

Hasegawa, A.: Plasma Instabilities and Nonlinear Effects, SpringerVerlag, Berlin, 1975.

$\mathrm{Hu}$, Y. Q. and Habbal, S. R.: Resonant acceleration and heating of solar wind ions by dispersive ion cyclotron waves, J. Geophys. Res., 104, 17 045-17 056, 1999.

Huddleston, D. E., Strangeway, R. J., Warnecke, J., Russell, C. T., Kivelson, M. G., and Bagenal, F.: Ion cyclotron waves in the Io torus during the Galileo encounter: Warm plasma dispersion analysis, Geophys. Res. Lett., 24, 2143-2146, 1997.

ITER: Physics Expert Group on Energetic Particles, Heating and Current Drive and ITER Physics Basis Editors: Chapter 6: Plasma auxiliary heating and current drive, Nucl. Fusion, 39, 12, 2495-2539, 1999.

Karpman, V. I. and Washimi, H.: On the ponderomotive force of a highfrequency electromagnetic field in a dispersive medium, Zh. Eksp. Teor. Fiz., 71, 1010-1016, 1976 (The ponderomotive force of a high-frequency electromagnetic field in a dispersive medium, Sov. Phys. JETP, 44, 528-531, 1976).

Karpman, V. I. and Washimi, H.: Two-dimensional selfmodulation of a whistler wave propagating along the magnetic field in a plasma, J. Plasma Phys. 18, 173-187, 1977.

Karpman, V. I. and Stenflo, L.: Equations describing the interaction between whistlers and magnetosonic waves, Phys. Lett. A, 127, 2, 99-101, 1988.

Kourakis, I., and Shukla, P.K.: Modulated whistler wavepackets associated with density perturbations, Physics of Plasmas, 12, 1, 012902/1-6, 2005.

Kourakis, I. and Shukla, P. K.: Exact theory for localized envelope modulated electrostatic wavepackets in space and dusty plasmas, Nonlin. Proc. Geophys., in press, 2005.

Lee, K. F.: A note on the bandwidths of electromagnetic ion cyclotron harmonic waves in plasmas, J. Appl. Phys., 434, 35863587, 1972.

Li, X., Habbal, S. R., Hollweg, J. V., and Esser, R.: Heating and cooling of protons by turbulence-driven ion cyclotron waves in the fast solar wind, J. Geophys. Res., 104, 2521-2535, 1999.

Lund, E. J. and LaBelle, J.: On the generation and propagation of auroral electromagnetic ion cyclotron waves, J. Geophys. Res., 102, 17 241-17 253, 1997.

Mazelle, C., Winterhalter, D., Sauer, K., Trotignon, J. G., Acuña, M. H., Baumgärtel, K., Bertucci, C., Brain, D. A., Brecht, S. H., Delva, M., Dubinen, E., Oieroset, M., and Slavin, J.: Bow Shock and Upstream Phenomena at Mars, Space Science Reviews, 111, 115-181, 2004.

McFadden, J. P., Carlson, C. W., Ergun, R. E., Chaston, C. C., Mozer, F. S., Temerin, M., Klumpar, D. M., and Shelley, E. G.: Electron modulation and ion cyclotron waves observed by FAST, Geophys. Res. Lett., 25, 12, 2045-2048, 1998.

Meziane, K., Mazelle, C., Wilber, M., LeQuéau, Eastwood J.P., Rème, H., Dandouras, I., Sauvaud, J. A., Bosqued, J. M., Parks, G. K., Kistler L. M., McCarthy, M., Klecker, B., Korth, A., Bavassano-Cattaneo, M. B., Lundin, R. N., and Balogh, A.: Bow shock specularly reflected ions at the presence of low-frequency 
electromagnetic waves: a case study, Ann. Geophys., 22, 23252335, 2004a,

SRef-ID: 1432-0576/ag/2004-22-2325.

Meziane, K., Wilber, M., Mazelle, C., LeQuéau, D., Kucharek, H., Lucek, E.A., Rème, H., Hamza, A. M., Sauvaud, J. A., Bosqued, J. M., Dandouras, I., Parks, G. K., McCarthy, M., Klecker, B., Korth, A., Bavassano-Cattaneo, M. B., and Lundin, R. N.: Simultaneous observations of field-aligned beams and gyrating ions in the terrestrial foreshock, J. Geophys. Res. (Space Phys.), 109, A05, A05107, 2004b.

Pottelette, R., Ergun, R. E., Treumann, R. A., Berthomier, M., Carlson, C. W., McFadden, J. P., and Roth, I.: Modulated electronacoustic waves in auroral density cavities: FAST observations, Geophys. Res. Lett., 26, 16, 2629-2632, 1999.

Praburam, G., Tripathi, V. K., and Mishra, G.: Nonlocal theory of rf suppression of current-driven ion cyclotron waves in a Q machine, Phys. Rev. A, 43, 968-972, 1991.

Rao, N. N. and Shukla, P. K.: Coupled Langmuir and ion-acoustic waves in two-electron temperature plasmas, Phys. Plasmas, 4, 636-645, 1997.

Rao, N. N. and Shukla, P. K.: Coupled whistler and ion-acoustic mode propagation in two-electron-temperature plasmas, Phys. Lett. A, 243, 151-155, 1998.

Remoissenet, M.: Waves called solitons, Springer-Verlag, Berlin, 1994.

Russell, C. T., Kivelson, M. G., Kurth, W. S., and Gurnett, D. A.: Depleted magnetic flux tubes as probes of the Io torus plasma, Adv. Space Res., 28, 10, 1489-1493, 2001.
Santolik, O., Gurnett, D. A., Pickett, J. S., Parrot, M., and Cornilleau-Wehrlin, N.: Spatio-temporal structure of storm-time chorus, J. Geophys. Res., 108, 1278/1-14, 2003.

Shukla, P. K. and Stenflo, L.: Nonlinear propagation of electromagnetic waves in magnetized plasmas, Phys. Rev. A, 30, 2110 2112, 1984.

Shukla, P. K. and Stenflo, L.: Nonlinear propagation of electromagnetic ion-cyclotron Alfvén waves, Phys. Fluids, 28, 5, 1576$1578,1985$.

Shukla, P. K. and Stenflo, L.: Growth rates of modulationally unstable ion-cyclotron Alfvén waves, Phys. Scripta 34, 169-170, 1986.

Shukla, P. K., Stenflo, L., Bingham, R., and Eliasson, B.: Nonlinear effects associated with dispersive Alfvén waves in plasmas, Plasma Phys. Cont. Fusion, 46, B349-358, 2004.

Stasiewicz, K., Bellan, P. M., Chaston, C. C., Kletzing, C., Lysak, R., Maggs, J., Pokhotelov, O., Seyler, C., Shukla, P. K., Stenflo, L., Streltsov, A., and Wahlund, J.-E.: Small scale Alfvénic structure in the aurora, Space Sci. Rev., 92, 423-533, 2000.

Stenflo, L., Shukla, P. K., and Yu, M. Y.: Nonlinear propagation of electromagnetic waves in magnetized electron-positron plasmas, Astrophys. Space Sci., 117, 303-308, 1985.

Stenzel, R. L.: Whistler waves in space and laboratory plasmas, J. Geophys. Res., 104, A7, 14 379-14 395, 1999.

Stix, T.: Waves in Plasmas, American Institute of Physics, New York, 1992.

Sulem, C., and Sulem, P. L.: The Nonlinear Schrödinger Equation, Springer, Berlin, 1999. 